See discussions, stats, and author profiles for this publication at: https://www.researchgate.net/publication/324855049

\title{
Multiple Tolerization Reduces Antibody Binding Against Tolerogen Cells: Implications for the Production of Monoclonal Antibodies
}

Article in Monoclonal Antibodies in Immunodiagnosis and Immunotherapy · April 2018

DOI: $10.1089 / \mathrm{mab} .2017 .0055$

CITATIONS

0

8 authors, including:

Rodrigo Almeida

São Paulo State University

4 PUBLICATIONS 35 CITATIONS

SEE PROFILE

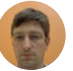

Matheus Bertanha

São Paulo State University

38 PUBLICATIONS 42 CITATIONS

SEE PROFILE

Some of the authors of this publication are also working on these related projects:

stem cell project View project

RED LED OF bioestimulation EFFECT OF CONTINUOUS ASSESSMENT AND pulsatile IN CELL CULTURE OF STEM CELLS MESENCHYMAL. View Project
READS

48

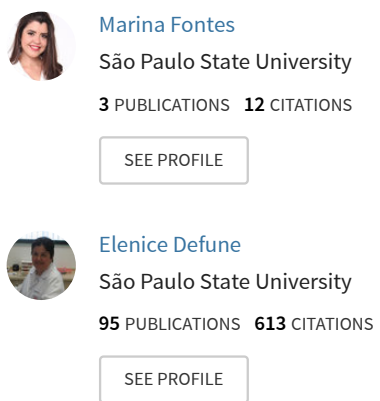




\title{
Multiple Tolerization Reduces Antibody Binding Against Tolerogen Cells: Implications for the Production of Monoclonal Antibodies
}

\author{
Rodrigo de Almeida, Cecília Naomi Nakamura,' Marina de Lima Fontes, Julia Paiola da Silva, \\ Matheus Bertanha, Elenice Deffune, Ana Marisa Fusco-Almeida, and Andrei Moroz ${ }^{1-3}$
}

\begin{abstract}
We report an immunization technique that can update the production of monoclonal antibodies (mAbs): the multiple tolerization subtractive immunization (MTSI). A total of $10 \mathrm{BALB} / \mathrm{C}$ mice were used. Animals in group 1 received one inoculation of RWPE-1 cells (nontumoral), followed by cyclophosphamide, and then received serial inoculations of nonirradiated PC3 cells (tumoral). Animals in group 2 received our MTSI protocol, as follows: one inoculation of RWPE-1 cells, followed by cyclophosphamide (Cy). This whole tolerization step was repeated three other times, with 14-day intervals between the last $\mathrm{Cy}$ exposure and the next RWPE-1 cell inoculation. Finally, the animals received the same nonirradiated PC3 cell exposure as group 1. Blood was taken from each animal, and their polyclonal sera individually tested against the nontumoral RWPE-1 cells in flow cytometry. We found out that, after the MTSI was employed, the serum of the immunized animals, in group 2, contained considerably less antibodies that reacted against the tolerogenic cells, compared with the serum of the animals that underwent regular subtractive immunization. We showed that, by repeating the tolerization cycles, the polyclonal antibodies produced by mice have a reduced specificity toward common/immunodominant epitopes present at nontumoral cells, and thus this technique can be readily used by others in studies involving murine mAb protocols.
\end{abstract}

Keywords: subtractive immunization, monoclonal antibodies, murine, hybridoma, immunization, tolerization

B IOFABRICATED CELL/PROTEIN biosensors have been areas of intense research for some years now. Interestingly, this high-tech platform relies on the use of a very old tool, the monoclonal antibody (mAb), although it has been more than four decades since Köhler and Milstein described the technology of hybridomas as permanent biofactories. ${ }^{(1)}$ Their findings paved the way for the production of mAbs, powerful research tools that have revolutionized biomedical science in several areas, including cancer research, stem cell biology, and now, biosensors. Despite the growing advances in technology (e.g., engineered human antibodies, diabodies, transgenic mice platforms, phage display), classical murine hybridoma technology can still deliver unique antibodies for research and development. Producing antibodies that may recognize novel biomarkers critical to understanding some pathological conditions is just one possible use of the technology.

An old strategy such as murine hybridoma $\mathrm{mAb}$ production, however, requires some upgrading and tweaking to fulfill the abovementioned promise. The question is how? Because we rely on this technology, our laboratory has been searching for alternatives to renew $\mathrm{mAb}$ production. After a considerable study, we have concluded that the best manner to update, tweak, and renew is to employ a subtractive immunization with some modifications, described below, when producing mAbs through hybridomas. $^{(2-4)}$

To understand why, we must first understand this technique. Described in the early 1990s, subtractive immunization is a procedure where two sets of immunogenic substances are employed (tolerogen and immunogen) intercalated with cyclophosphamide (Cy)..$^{(2,3)}$ Tolerogen and immunogen must be similar, but differ only in some selected properties. One example, and perhaps the best one, is when one desires mAbs that react only against tumor-associated antigens. In this case, cells from the same tissue origin are used in their nontumoral and tumoral phenotypes as the tolerogen and immunogen, respectively. ${ }^{(2,3)}$ Other techniques include neonatal

\footnotetext{
${ }^{1}$ Monoclonal Antibody Laboratory, Proteomics Center, Univ Estadual Paulista-UNESP, School of Pharmaceutical Sciences, Araraquara, Brazil.

${ }^{2}$ Cell Engineering Laboratory, Blood Transfusion Center, Univ Estadual Paulista-UNESP, School of Medicine, Botucatu, Brazil.

${ }^{3}$ Department of Bioprocess and Biotechnology, Univ Estadual Paulista-UNESP, School of Pharmaceutical Sciences, Araraquara, Brazil.
} 
tolerization and high-zone tolerance. For a broader view of these techniques, investigators are invited to read our review on this theme. ${ }^{(4)}$ Accumulated evidence has shown that this approach is able to actively modify the biological behavior of the immune response within mice, directing it against rare antigens, such as those that directly influence tumoral cell metastasis or other relevant biological processes. In this sense, several reports have demonstrated the feasibility of this technique to provide $\mathrm{mAbs}$ specific for tumors in liver, pancreas, lung, and other tumors. ${ }^{(5-8)}$

Our group has also verified the efficacy of this technique during a mAb production protocol that has delivered some promising $\mathrm{mAbs}$ against human prostate tumor cells that do not crossreact with their prostate nontumoral counterparts. However, we also observed that a considerable number of hybridoma cell lines were still producing antibodies that did react against the tolerogen cells (unpublished data). Therefore, we concluded that the subtractive immunization technique, as classically described by Williams et al. and Brooks et al., ${ }^{(2,3)}$ has only limited efficacy in yielding highly specific antibodies. This prompted us to look further for more positive results.

While investigating why antibodies reacted with nontumoral cells, (probably through immunodominant shared epitopes), we found a report by Jin et al., ${ }^{(9)}$ in which the authors employed subtractive immunization and produced antibodies that were specific to a limited strain of enterohemorrhagic bacteria, the Escherichia coli O157:H7, (a common contaminant of food, which causes subsequent diarrhea). These mAbs were highly specific, and no crossreactivity with other enterobacteria was observed. ${ }^{(9)}$ However, what immediately caught our attention was the fact that these researchers, unlike others, performed additional tolerization steps sequentially before introducing the actual immunogen in the host. Searching for similar reports, we could not find any that used multiple tolerization to produce antitumor antibodies. We then modified our immunization protocols and performed the following experiment.

All animal manipulation respected the Guidelines of Animal Experimentation approved by the Commission of Ethics in Animal Manipulation (Protocol number CEUA/FCF/CAr $\left.n^{\circ} 22 / 2015\right)$ at the School of Pharmaceutical Sciences, UNESP. We used ten 5-week-old BALB/C male mice in our experiments, divided into two different groups for immunization: Group 1-Single tolerization (may also be defined as subtractive immunization), and Group 2-Multiple tolerization (may also be defined as multiple tolerization subtractive immunization [MTSI]). Animals in group 1 received one inoculation of nonirradiated RWPE-1 cells (human prostate, epithelial, nontumoral, $2 \times 10^{6} / \mathrm{animal}$ ), followed by $\mathrm{Cy}$ (200 mg/kg, 10 minutes, 24 hours, and 48 hours following RWPE-1 cells) and then received serial inoculations of nonirradiated PC3 cells (human prostate, epithelial, tumoral, $2 \times 10^{6}$ cells/animal, three times in 14-day intervals).

Animals in group 2 received our MTSI protocol, as follows: one inoculation of nonirradiated RWPE-1 cells (human prostate, epithelial, nontumoral, $2 \times 10^{6} / \mathrm{animal}$ ), followed by Cy $(200 \mathrm{mg} / \mathrm{kg}, 10$ minutes, 24 hours, and 48 hours following RWPE-1 cells). This whole tolerization step was repeated three other times, with 14-day intervals between the last $\mathrm{Cy}$ exposure and the next RWPE-1 cell inoculation. Therefore, group 2 received multiple tolerization/immunosuppression rounds. Finally, the animals received the same nonirradiated
PC 3 cell exposure as group 1. All of these immunizations were performed intraperitoneally (IP). No adjuvant was used. Cy was also administered IP. At the end, animals were euthanized for hybridoma production (data not shown/ underway).

Also, blood was taken from each animal, 1 day before being euthanized, and their sera individually tested against the nontumoral RWPE-1 cells in flow cytometry. Each serum was individually diluted 1:200 in ISOTON ${ }^{\mathrm{TM}}$ and then incubated with $1 \times 10^{5}$ RWPE- 1 cells for 2 hours. Unbounded antibodies were washed away and a secondary antibody (goat anti-mouse $\operatorname{IgG}+\operatorname{IgM}$ ), coupled with FITC, was added to the tubes (also diluted 1:200 in ISOTON). Secondary antibody was incubated during 1 hour and a half. Unbounded antibodies were washed away and the events were captured. The population gate was defined and positive counts (cells with antibody binding) were recorded with FACS Canto ${ }^{\mathrm{TM}}$. Positive controls (anti-CD81, data not shown) and isotype controls (anti-CD86; nonimmune serum from other mice) were also used. These controls were chosen based on an extensive investigation of the phenotype of human prostate cell lines, regarding cell surface antigens. ${ }^{(10)}$ Positive results from each animal were analyzed per group, using the Instat ${ }^{\mathrm{TM}}$ software, with a nonpaired Student's $T$ test, comparing the different immunization protocols by the mean of positive cells.

Our finding was that, after the MTSI was employed, the serum of the immunized animals, in group 2, contained considerably less antibodies (mean of $\sim 52 \%$ positive counts; $20.5 \% \mathrm{SD} ; 39.13 \% \mathrm{CV}$ ) that reacted against the tolerogen (RWPE-1 cells), as an initial observation, compared with the serum of the animals that underwent regular subtractive immunization (group 1), with only one round of tolerization/ immunosuppression (mean of $\sim 98 \%$ positive counts; $\pm 2.47 \%$ $\mathrm{SD} ; \pm 2.53 \% \mathrm{CV}$ ) (Fig. 1). This result was statistically significant $(p<0.05)$. Positive and isotype controls worked as expected. Cell fusions are being screened now, but we believe that the frequency of hybridoma cell lines producing mAbs that reacts against the immunogen will be higher, whereas the frequency of hybridoma cell lines producing mAbs that reacts against the tolerogen should definitely decrease.

But how does the MTSI work? We believe that when each step of tolerization is performed, a percentage of the antigens anchored to the cell membrane is processed, resulting in the selection of clones that are specific to those antigens (Fig. 2). We also believe this percentage to be small/limited, and therefore, when multiple steps of tolerization are repeated, increasing amounts of antigens are processed resulting in a larger coverage of the antigens anchored to the cell membrane. Since we employ cyclophosphamide after the tolerogen is inoculated, all the proliferating lymphocytes should be eliminated. Other mechanism of action could be through the elimination of helper $\mathrm{T}$ cells, required for B cell maturation and differentiation. Also, memory B cells are eliminated, which nullifies the effects of a possible second encounter with a previously exposed antigen. Given that one B cell can only produce one antibody (in terms of specificity to a single epitope), each tolerization step removes a pool of antigenspecific B cells from the animals' immune system repertoire (naive B cells). Therefore, we think that these multiple steps of tolerization are beneficial to a more complete tolerization of the immunodominant epitopes. Finally, given that tolerogen and immunogen derives from the same tissue origin, and 


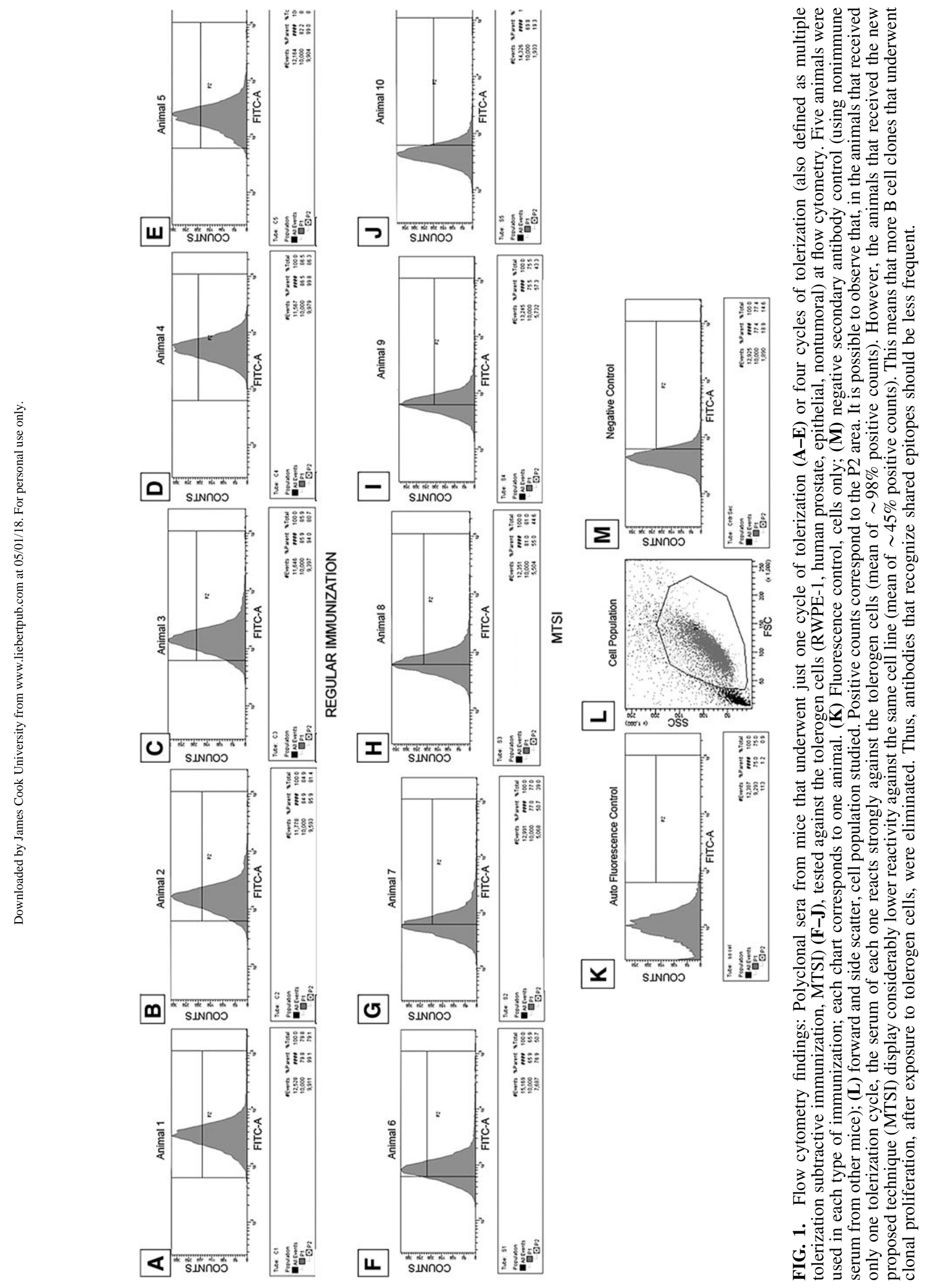




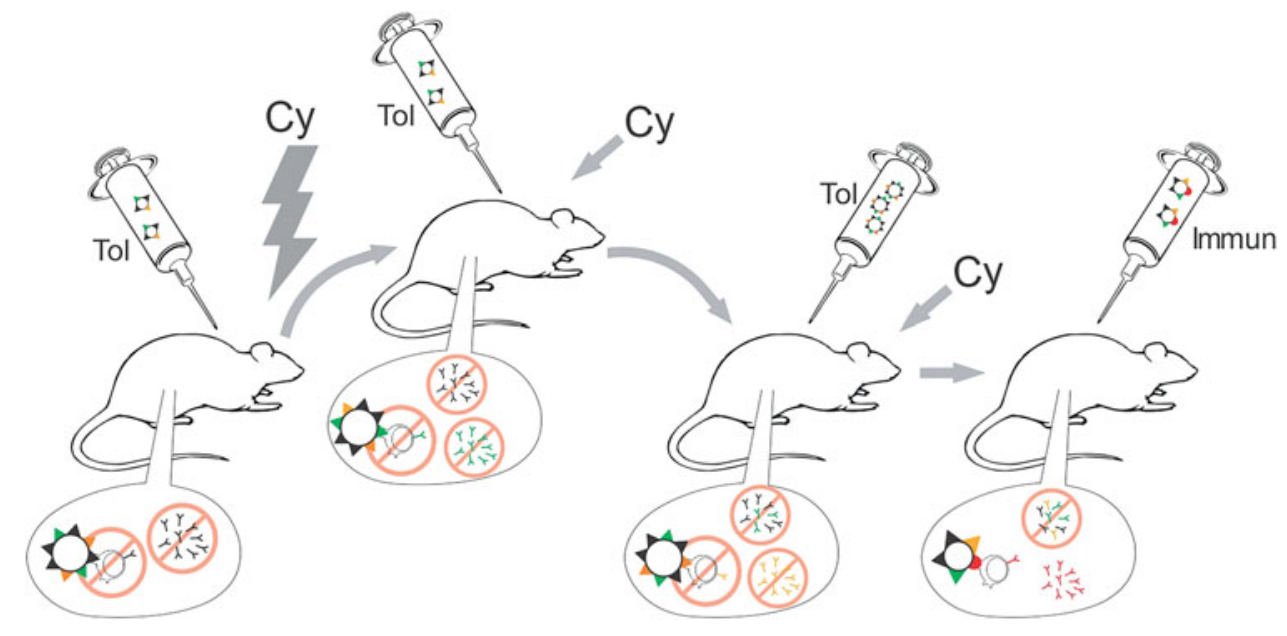

FIG. 2. MTSI and how we propose it works: In this technique, described by our group, adult mice (6 weeks old) are initially administered tolerogen cells. The set of antigens anchored to cell membranes (represented as black/orange/green triangles) would trigger a humoral reaction; however, following 24 and $48 \mathrm{~h}$, the same mice are given cyclophosphamide (Cy), typically at $200 \mathrm{mg} / \mathrm{kg}$. This immunosuppressive drug eliminates B cells that underwent clonal selection and expansion; triggered by the previous exposure to the tolerogen antigens (in this case, black triangles). It is important to notice that neither B cells nor memory cells are produced. However, and here is the central argument, the tolerogen cells present far too many antigens for the host's immune system to process in a single exposure. In this sense, only a limited portion of antigens really becomes tolerized (again, represented as black triangles). The tolerization/immunosuppression steps are repeated sequentially and, in each, a different set of antigens will be processed by the host's immune system (represented as green triangles, followed by orange triangles). We believe that, after a number of repetitions, a higher percentage of the tolerogen antigens indeed becomes tolerized. Finally, the same animals are given immunogen cells, which are similar to tolerogen cells (e.g., same tissue origin), however, they present distinct characteristics (e.g., tumor-associated antigens, represented as red dots). Shared antigens/epitopes (represented as black/orange/green triangles) were already tolerized (by the elimination of B cells, induced by $\mathrm{Cy}$ ) and does not trigger a humoral reaction. B cells specific to rare/low-expression antigens (red) undergo clonal selection, resulting in specific monoclonal antibody production.

both are epithelial cells, a large percentage of these immunodominant epitopes should be shared, resulting in a specific immune response to the epitopes that are expressed only by the immunogen cells.

In addition to cancer research, another area that may benefit from highly specific mAbs produced with this novel technique is adult stem cell biology. Currently, the availability of engineered tissue for regenerative medicine is limited because it is difficult to correctly identify all of the different populations of stem cells available and their actual plasticity, given that these cells share numerous antigens. For example, it has been reported that there are more than 10 different phenotypes of mesenchymal stem cell-like populations and others in the bone marrow alone. ${ }^{(11)}$ The difficulties arise from the fact that, to date, mAbs that can easily and specifically identify these different cell lines are not available. We hypothesize that this is because the antibodies under use recognize epitopes, which may be shared with other stem cell niches or other cell types, resulting in difficulty to definitively characterize the cells. We also believe that using some of these lines, in different combinations of tolerogens/immunogens using the MTSI, would be an efficient manner to obtain a panel of mAbs that can correctly and definitively identify them.

It must be noted that there are some potential drawbacks on this technique. For example, it was hypothesized that cyclophosphamide not only eliminates the clones of B cells in proliferation, which would generate antibodies reactive with normal cell antigens from the tolerogen (desired effect), but that this drug also eliminates helper $\mathrm{T}$ cells required for $\mathrm{B}$ cell maturation and differentiation (undesired effect, when the immunogen is injected). Therefore, this could lead to a scenario in which, when the animals receive the immunogen, only lowaffinity antibodies of the IgM isotype could be retrieved. ${ }^{(12)}$ To prevent this undesired effect, we must take into account that some time is required for the host to start to produce cells that will differentiate into helper $\mathrm{T}$ cells. Therefore, the inoculation of the immunogen should be at least 21 days after the last dose of cyclophosphamide is performed. It is also very important to constantly titer the polyclonal antibodies in mice sera against the tolerogen as well as screen the mice for cytotoxic effects due to cyclophosphamide exposure.

Finally, we also hypothesize that combining different approaches of subtractive immunization could be an interesting way to obtain novel mAbs. For example, neonatal tolerization with cyclophosphamide-induced tolerization would prevent the undesirable production of antibodies that recognize immunodominant epitopes from the tolerogen. Another strategy would be the production of polyclonal antibodies (against the tolerogen) in mice, before the subtractive immunization with cyclophosphamide. Then, during this immunization, other mice that are being immunized with the tolerogen could also receive, in that same time, the polyclonal antibodies produced earlier (masking immunization technique). In this scenario, the production of undesirable antibodies against the tolerogen would be blocked in two different axes, that is, by the polyclonal antibodies and druginduced lymphocyte death.

In conclusion, by repeating the tolerization cycles we have demonstrated that the polyclonal antibodies produced by mice 
have a reduced specificity toward common/immunodominant epitopes present at nontumoral cells. We believe that one can obtain novel mAbs using a classical and well-assimilated technique such as murine hybridomas, by making simple adjustments of the immunization scheme. MTSI does not require any other modifications other than those described above, and thus this technique can be readily used by others in studies involving murine $\mathrm{mAb}$ protocols.

\section{Acknowledgments}

The authors would like to thank Mr. Christopher Gieseke at the University of Texas at San Antonio, and Mr. Sebastian Locksenius at the University of Stockholm, for providing excellent assistance in the English language revision of this article. Fundação de Amparo à Pesquisa do Estado de São Paulo (FAPESP) (processes n ${ }^{\circ}$ 2014/23738-8, 2015/21884-0, 2016/07047-0) and Conselho Nacional de Desenvolvimento Científico e Tecnológico (CNPq) (process n $\left.{ }^{\circ} 425535 / 2016-9\right)$ are also greatly acknowledged for research fund grants.

\section{Author Disclosure Statement}

No competing financial interests exist.

\section{References}

1. Köhler G, and Milstein C: Continuous cultures of fused cells secreting antibody of predefined specificity. Nature 1975;256:495-497.

2. Williams CV, Stechmann CL, and McLoon SC: Subtractive immunization techniques for the production of monoclonal antibodies to rare antigens. Biotechniques 1992;12:842-847.

3. Brooks PC, Lin JM, French DL, and Quigley JP: Subtractive immunization yields monoclonal antibodies that specifically inhibit metastasis. J Cell Biol 1993;122:1351-1359.

4. de Almeida R, Nakamura CN, de Lima Fontes M, Deffune E, Felisbino SL, Kaneno R, Fávaro WJ, Billis A, Cerri MO, Fusco-Almeida AM, Mendes Giannini MJ, and Moroz A: Enhanced immunization techniques to obtain highly specific monoclonal antibodies. mAbs 2018;10:46-54.

5. Boukerche H, Baril P, Tabone E, Bérard F, Sanhadji K, Balme B, Wolf F, Perrot $\mathrm{H}$, and Thomas L: A new $\mathrm{Mr}$ 55,000 surface protein implicated in melanoma progression:
Association with a metastatic phenotype. Cancer Res 2000; 60:5848-5856.

6. Hooper JD, Zijlstra A, Aimes RT, Liang H, Claassen GF, Tarin D, Testa JE, and Quigley JP: Subtractive immunization using highly metastatic human tumor cells identifies SIMA135/CDCP1, a $135 \mathrm{kDa}$ cell surface phosphorylated glycoprotein antigen. Oncogene 2003;22:1783-1794.

7. Rasmussen N, and Ditzel HJ: Scanning the cell surface proteome of cancer cells and identification of metastasisassociated proteins using a subtractive immunization strategy. J Proteome Res 2009;8:5048-5059.

8. Yasumoto M, Hamabashiri M, Akiba J, Ogasawara S, Naito Y, Taira T, Nakayama M, Daicho A, Yamasaki F, Shimamatsu K, Ishida Y, Kaji R, Okabe Y, Nakashima O, Ohshima K, Nakashima M, Sata M, and Yano H: The utility of a novel antibody in the pathological diagnosis of pancreatic acinar cell carcinoma. J Clin Pathol 2012;65:327-332.

9. Jin M, Lang J, Shen ZQ, Chen ZL, Qiu ZG, Wang XW, and Li JW: A rapid subtractive immunization method to prepare discriminatory monoclonal antibodies for food E. coli O157: H7 contamination. PLoS One 2012;7:1-7.

10. Liu AY: Differential expression of cell surface molecules in prostate cancer cells. Cancer Res 2000;60:3429-3434.

11. Ratajczak MZ, Zuba-Surma EK, Machalinski B, and Kucia M: Bone-marrow-derived stem cells-our key to longevity? J Appl Genet 2007;48:307-319.

12. Bowdish KS, Xin H, Maruyama T, and Dakappagari N: Antibodies against cancer produced using masked cancer cells as immunogen. Patent Application number EP20050771329 20050708, 2007, United States of America.

Address correspondence to: Andrei Moroz Monoclonal Antibody Laboratory Univ Estadual Paulista-UNESP School of Pharmaceutical Sciences Proteomics Center Araraquara 14800-903 Brazil

E-mail: moroz@fcfar.unesp.br

Received: October 5, 2017 Accepted: March 7, 2018 